\title{
ANTHROPOLOGICAL PICTURE OF THE CORDED WARE POPULATION OF THE SUBCARPATHIAN REGION IN THE LIGHT OF DATA OBTAINED FROM THE SITES AT ŚWIĘTE, JAROSŁAW DISTRICT
}

\begin{abstract}
Twenty Corded Ware graves containing twenty five interments were identified at sites 11, 15 and 20 at Święte, Radymno Commune, Jarosław District. In most of the graves (16), there was only one interment, although there were also three graves with two internments and one grave with three interments. The age and sex structure of buried individuals shows the roughly equal number of females, males, and children at the age of Infans I and II, with the category Maturus (individuals aged 40 to 50) having the highest mortality rate. So far, Rzeszów Foothills and the Lower San River Valley yielded 40 Corded Ware graves containing 51 interments in total. Single burials were predominant. Alike at Święte sites, the highest proportion of burials is recorded for individuals at the age of Maturus irrespective of sex; overall, for the entire region, male graves, however, outnumber female graves. In most cases skeletons are poorly preserved, but scarce anthropometric data are the indication of dolichomorphic crania in both males and females, which phenomenon is already noted for the Małopolska Upland and present Ukraine. The intravital body height
\end{abstract}

ABSTRACT

* Institute of Archaeology and Ethnology, Polish Academy of Sciences, Sławkowska 17, 31-016 Kraków, Poland; Department of Anatomy, Kopernika 12, 31-034 Kraków, Poland; anita.szczepanek@uj.edu.pl 
for adults varies from 161.3 to $175 \mathrm{~cm}$ for males and from 156.7 to $163.1 \mathrm{~cm}$ for females.

Key words: Corded Ware culture, Święte, anthropological analysis, Subcarpathian region

Research work done prior to construction of the A4 motorway between Rzeszów and Korczowa in the first decade of the $21^{\text {st }}$ century yielded considerable amounts of materials attributable to the Corded Ware culture (CWC). As is the case for other CWC settlement areas, the materials almost exclusively comprise sepulchral features, including specifically niche graves. In addition to graves at sites 11, 15 and 20 at Święte, Jarosław District, burials were also identified at sites 24 and 27 at Mirocin, Przeworsk District [Szczepanek 2019]; Rozbórz, Przeworsk District [Szczepanek 2013a]; Chłopice, Jarosław District [Szczepanek 2012]; Skołoszów, Jarosław District [Szczepanek 2013b]; and Szczytna, Jarosław District [Rogóż 2017; Szczepanek 2017]. Earlier research work in the 1970s brought to light just one CWC burial that was most likely found in the niche grave at site 81 in Przemyśl [Koperski 1973; Szczepanek 2004], with a disturbed postcranial skeleton being the only preserved element.

Anthropological analyses covering the sites at Święte were performed by W. Kozak-Zychman (site 11) [Kozak-Zychman 2011], P. Dąbrowski (site 20) [Dąbrowski 2012] and H. Rutka (site 15) [Rutka 2013], with results enclosed with relevant archaeological reports for the sites. In total, twenty graves identified at the sites at Święte contained the remains of twenty five individuals, whose age and sex demographic is shown in Table 1. In several cases, data obtained by genetic testing (aDNA) are included.

In most of the graves (16), there was only one interment, although there were also three graves with two internments and one grave with three interments. The age and sex structure of the buried individuals shows the roughly equal number of females, males, and children at the age of Infans I and II, with the category Maturus (individuals aged 40 to 50 ) having the highest mortality rate.

The analysis of bone pathologies revealed them being typical of mature adults, who constitute the largest group within the examined set of individuals. Classified to the age group Maturus, the individuals were suffering from dental caries predominantly affecting molars, as well as from frequent intravital tooth loss which tends to be the result of pre-existing conditions. Obliteration of alveolar bones of molars was identified in the male from grave 876, site 11 (left maxillary and mandibular M2s and right maxillary M3) and in the male of the age group Senilis from grave1149, site 11 (left maxillary M2). Moreover, extensive caries affecting molars (right maxillary M2 and right and left maxillary M1s) was detected in the male from grave 876, site 11. The female of the age group Adultus from grave $40 \mathrm{a}$, site 15 , was found to have small bone fistulas affecting the vicinity of roots of left maxillary teeth, caused by alveolar osteitis. The identified dental pathologies 
Individuals buried in the graves at the sites of Święte, Jarosław Distric

\begin{tabular}{|c|c|c|c|c|c|}
\hline Grave number & $\begin{array}{l}\text { Number of individuals } \\
\text { in grave }\end{array}$ & $\begin{array}{l}\text { Age } \\
\text { (category) }\end{array}$ & $\begin{array}{l}\text { Age } \\
\text { [years] }\end{array}$ & Sex & aDNA \\
\hline \multicolumn{6}{|l|}{ Święte, site 11} \\
\hline 756 A part $\mathrm{N}$ & \multirow[t]{2}{*}{2} & $?$ & $?$ & $?$ & \\
\hline 756 A part $S$ & & Infans I / II & $7-8$ & $?$ & \\
\hline 791 & 1 & adult? & $20-x$ & $\mathrm{~F}$ ? & \\
\hline 814 & 1 & Maturus & $40-50$ & $?$ & \\
\hline $863 \mathrm{~A}$ & 1 & adult & $20-x$ & $?$ & \\
\hline 863B & 1 & Adultus & $20-30$ & $\mathrm{~F}$ & \\
\hline 876 & 1 & Maturus & $30-35$ & $\mathrm{M}$ & $\mathrm{XY}$ \\
\hline 1134 & 1 & Adultus & $25-30$ & M & \\
\hline 1149 & 1 & Senilis & $60-65$ & M & $\mathrm{XX}$ ? \\
\hline $1290 \mathrm{D}$ & 1 & Adultus & $25-30$ & M & $\mathrm{XY} ?$ \\
\hline 1434 & 1 & Maturus & $40-50$ & $\mathrm{~F}$ & \\
\hline \multicolumn{6}{|l|}{ Święte, site 15} \\
\hline 173 & 1 & Maturus? & $40-50$ & $\mathrm{~F}$ & $\mathrm{XX}$ ? \\
\hline $405 \mathrm{~A}$ & 1 & adult & $20-x$ & $?$ & \\
\hline 407 & 1 & Maturus & $40-50$ & $\mathrm{~F}$ & \\
\hline $408 \mathrm{~b}$ & \multirow[t]{2}{*}{2} & Adultus/Maturus & $30-40$ & M & $\mathrm{XX}$ \\
\hline $408 \mathrm{a}$ & & Adultus & 20-30 & $\mathrm{F}$ & $\mathrm{XX}$ \\
\hline 409 & 1 & adult & $20-\mathrm{x}$ & M & \\
\hline \multirow[t]{2}{*}{423} & \multirow[t]{2}{*}{2} & Infans I & $1.5-2$ & $?$ & \\
\hline & & Infans I & $1.5-2$ & $?$ & \\
\hline 427 & 1 & Infans I & $5-6$ & $?$ & \\
\hline $431 \mathrm{~A}$ & 1 & Maturus & $40-50$ & M & \\
\hline \multicolumn{6}{|l|}{ Święte, site 20} \\
\hline $40 \mathrm{~A}$ & 1 & Infans II & $7-9$ & M? & $\mathrm{XY}$ \\
\hline \multirow[t]{3}{*}{43} & \multirow[t]{3}{*}{3} & Maturus & $40-45$ & M & $\mathrm{XY} ?$ \\
\hline & & Maturus & 44-55 & $\mathrm{F}$ & $\mathrm{XY}$ \\
\hline & & Infans II & $7-8$ & $?$ & \\
\hline
\end{tabular}


Ta ble 2

Rzeszów Foothills graves containing human remains

\begin{tabular}{|c|c|c|c|c|c|}
\hline Grave & $\begin{array}{l}\text { Number of } \\
\text { individuals } \\
\text { in grave }\end{array}$ & $\begin{array}{l}\text { Number of } \\
\text { individual }\end{array}$ & $\begin{array}{l}\text { Age } \\
\text { (category) }\end{array}$ & $\begin{array}{l}\text { Age } \\
\text { [years] }\end{array}$ & Sex \\
\hline \multicolumn{6}{|c|}{ Mirocin, site 24} \\
\hline 50 & 1 & & Maturus & $40-50$ & M \\
\hline \multirow[t]{2}{*}{53} & \multirow[t]{2}{*}{2} & I & adult & $20-x$ & $?$ \\
\hline & & II & Infans I & $3-4$ & $?$ \\
\hline 54 & 1 & & Senilis & $50-60$ & M \\
\hline \multirow[t]{2}{*}{110} & \multirow[t]{2}{*}{1} & I & Maturus & $40-50$ & M \\
\hline & & II* & Maturus & $40-50$ & M? \\
\hline \multicolumn{6}{|c|}{ Mirocin, site 27} \\
\hline 273 & 1 & & adult & $20-x$ & $?$ \\
\hline \multirow[t]{2}{*}{360} & \multirow[t]{2}{*}{2} & I & Senilis & $50-60$ & M \\
\hline & & II & Maturus & $40-50$ & $\mathrm{~F}$ \\
\hline 363 & $1 ?$ & & ? & $?$ & $?$ \\
\hline \multicolumn{6}{|c|}{ Szczytna, site 5} \\
\hline 217 & 1 & & Iuvenis & ok. 17 & $\mathrm{~F}$ \\
\hline \multirow[t]{2}{*}{220} & \multirow[t]{2}{*}{2} & I & Maturus & $40-50$ & M \\
\hline & & II & Maturus/Senilis & $45-55$ & M \\
\hline \multirow[t]{2}{*}{273} & \multirow[t]{2}{*}{2} & I & Infans II & $7-8$ & $?$ \\
\hline & & II & Infans II & $8-9$ & $?$ \\
\hline \multicolumn{6}{|c|}{ Szczytna, site 6} \\
\hline 2 & 1 & & Infans II/Iuvenis & $14-16$ & $?$ \\
\hline 4 & 1 & & Adultus & $20-30$ & $\mathrm{M}$ \\
\hline 56 & 1 & & Adultus & $24-30$ & M \\
\hline 84 & 1 & & Infans I & 6 & $?$ \\
\hline 150 & 1 & & Iuvenis & 15 & M \\
\hline \multicolumn{6}{|c|}{ Chłopice, site 26} \\
\hline \multirow[t]{2}{*}{11} & \multirow[t]{2}{*}{2} & I & Infans $\mathrm{II} /$ Iuvenis & $14-15$ & ? \\
\hline & & II & Infans II & $11-12$ & $?$ \\
\hline \multicolumn{6}{|c|}{ Rozbórz, site 42} \\
\hline 2006 & 1 & & Iuvenis/Adultus & $18-20$ & M \\
\hline
\end{tabular}




\begin{tabular}{|c|c|c|c|c|c|}
\hline \multirow[t]{2}{*}{256} & \multirow[t]{2}{*}{2} & $I^{*}$ & Infans II & $7-8$ & $?$ \\
\hline & & II & Adultus & $20-30$ & $\mathrm{~F}$ \\
\hline 620 & 1 & & Adultus & $20-30$ & M \\
\hline \multicolumn{6}{|c|}{ Przemyśl, site 81} \\
\hline & 1 & & adult & $20-x$ & M \\
\hline
\end{tabular}

*secondary burial of Mierzanowice Culture

$\mathrm{T}$ a b I e 3 Age and sex of individuals buried in graves containing two interments at the Rzeszów Foothills and in the Lower San River Valley

\begin{tabular}{|l|c|}
\hline Age and sex & Number of graves \\
\hline male and female & 2 \\
\hline subadult and subadult & 3 \\
\hline adult and subadult & 2 \\
\hline male and male & 1 \\
\hline subadult and undetermined & 1 \\
\hline
\end{tabular}

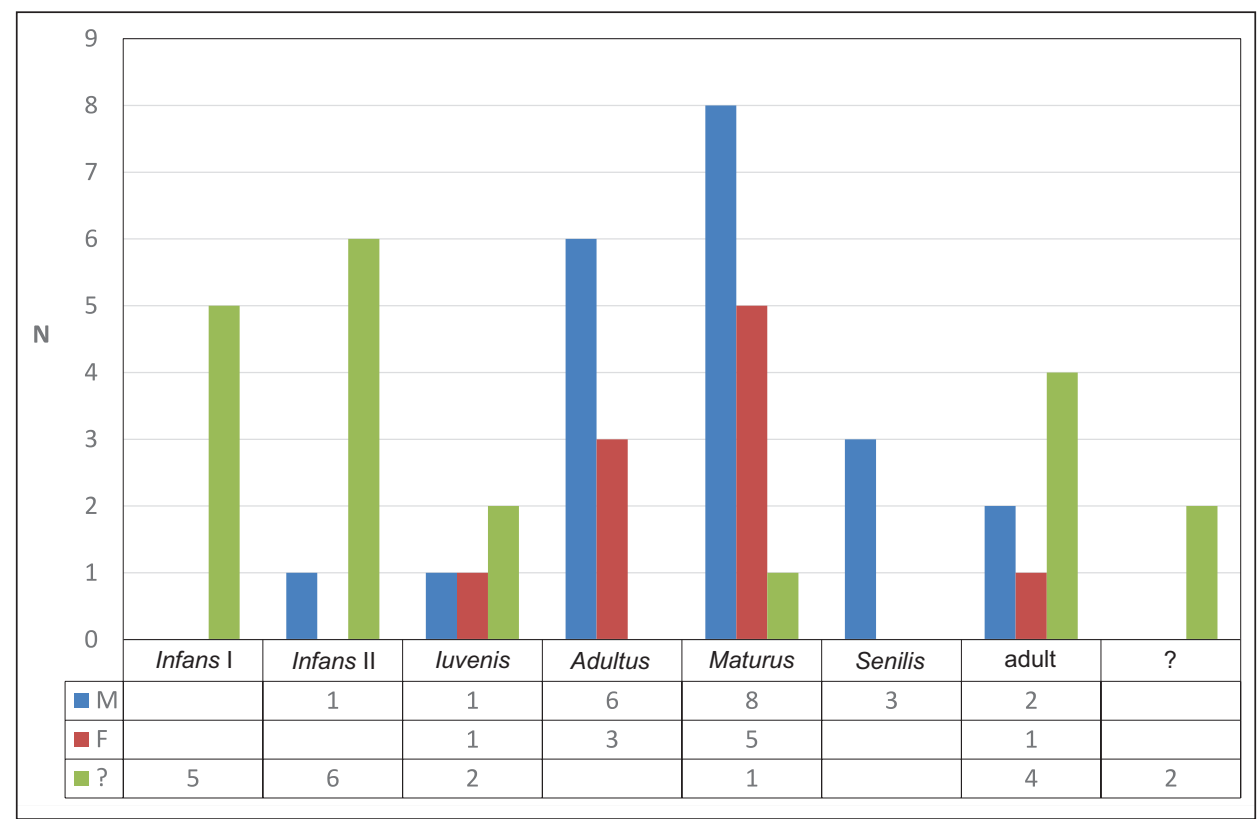

Fig. 1. Age and sex structure of Corded Ware culture individuals buried at the sites of the Rzeszów Foothills and Lower San River Valley 
prove that conditions occurred at a young age, and indirectly point to diets high in carbohydrates. Moreover, skeletal changes resulting from healed injuries were identified. This was the case for the male from grave 876 , site 11 , with a proximal femoral fracture leading to bone deformation and pseudoarthrosis on his pelvic bone. A right femoral shaft in the male of the age group Adultus from grave 1134, site 11, showed a bone overgrowth of unknown aetiology. A similar anomaly was identified in a right fibula of the male of the age group Adultus from grave 1290D, site 11. Roofs of eye sockets in children buried at site 15 exhibit a perforation cribra orbitalia which is a condition of complex aetiology most often attributable to anaemia of various origin [Walker et al. 2009].

Table 2 presents data concerning other CWC burials identified in the Rzeszów Foothills region. In total, the research work preceding construction of the A4 motorway provided data on 51 individuals found in 40 graves (20 features from Święte sites and 20 features from other areas of the Foothills).

As is the case for Święte, single burials predominate (14) at the Rzeszów Foothils sites. Moreover, six graves with two interments were found. The individuals buried in such graves were diversified in terms of age and sex (Table 3). Graves, in which two children were buried, are noteworthy for the fact that they always contain corpses of children of roughly similar age at death (Table 2).

The graph (Fig. 1) shows the age and sex structure of all CWC individuals so far identified in the Rzeszów Foothills and Lower San River Valley (sites at Święte) regions.

Mortality data for the Rzeszów Foothills and Lower San River Valley indicate prevalence of individuals belonging to the age group Maturus (40 to 50 years of age), irrespective of their sex. However, the number of males, who died in adulthood (Adultus), and children of the age group Infans I and II is roughly equal. What should be emphasized is that the male graves considerably outnumber female graves, with a male to female ratio being nearly $2: 1$. It is by no means easy to exactly say whether this is merely the reflection of the current state of scientific research or of purposeful preference for male burials.

Tables 4 and 5 show results of cranial measurements, and cranial indices obtained using those data. For purposes of comparison, data acquired for CWC people identified at various sites of the Małopolska Upland (Haduch 2008), averaged values for cemeteries at Żerniki Górne, Busko Zdrój District, and at Złota, Sandomierz District [Krenz-Niedbała 1999; 2000], as well as data for skeletons from the Lublin Upland were also used [Szczepanek 2016].

The obtaining of full metric characteristics was significantly hindered by the degree of skulls preservation: measurements of viscerocrania were possible in a few cases only. Absolute figures and converted cranial indices for males and females from the examined area reveal distinguishing properties of the examined group, including specifically long neurocrania as proved by large maximum cranial lengths $(g-o p)$. However, a small number of available measurements prevented any 
+
$\frac{0}{0}$
$\stackrel{0}{\circ}$
$\stackrel{5}{0}$

\begin{tabular}{|c|c|c|c|c|c|c|c|c|c|c|c|c|c|}
\hline 气̂: & 脊 & if & & & 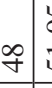 & $\frac{n}{\hat{n}}$ & $\begin{array}{l}\infty \\
\stackrel{g}{q} \\
\end{array}$ & 于 & in & 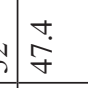 & $\stackrel{\infty}{+}$ & $\exists$ & F \\
\hline 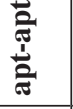 & $\stackrel{\Delta}{\Delta}$ & i & & & $\widehat{\approx}$ & $\begin{array}{l}\text { ¿े } \\
\text { तें }\end{array}$ & $\vec{n}$ & i & $\ddot{\sim}$ & $\overbrace{\vec{n}}^{m}$ & 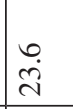 & 出 & $\overline{\mathrm{N}}$ \\
\hline & $\approx$ & & & & $m$ & $\begin{array}{l}\tilde{\infty} \\
\dot{m} \\
\dot{m}\end{array}$ & ले & r & $\bar{m}$ & $=\vec{m}$ & $\vec{m}$ & $\stackrel{\infty}{\sim}$ & 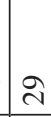 \\
\hline 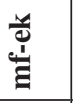 & F & & & & $q \oint$ & $\frac{\hat{\sigma}}{\dot{\gamma}}$ & $\begin{array}{l}\infty \\
\dot{q} \\
\dot{q}\end{array}$ & $\exists$ & I & $\begin{array}{l}\infty \\
\dot{m} \\
\dot{\gamma}\end{array}$ & $\begin{array}{l}\hat{a} \\
\infty \\
\infty\end{array}$ & $\stackrel{\infty}{m}$ & $m$ \\
\hline 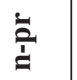 & 。̊. & $\begin{array}{c}\hat{0} \\
\dot{0}\end{array}$ & & & 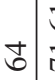 & $\begin{array}{l}\vec{b} \\
\dot{r}\end{array}$ & $\stackrel{9}{8}$ & 5 & ஜ & o & हे & $n$ & $\bar{n}$ \\
\hline 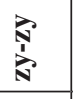 & & & & & 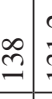 & $\frac{\stackrel{\sim}{M}}{\underline{m}}$ & $\stackrel{+}{\vec{m}}$ & $\stackrel{\infty}{\cong}$ & 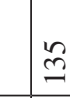 & 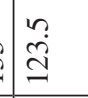 & $\stackrel{+}{\beth}$ & 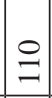 & 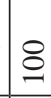 \\
\hline & & & $\stackrel{2}{9}$ & 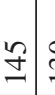 & 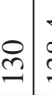 & & 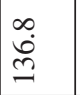 & 导 & I & خे & $\begin{array}{l}0 \\
\stackrel{1}{\mathrm{I}}\end{array}$ & $\stackrel{\infty}{\beth}$ & 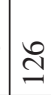 \\
\hline & 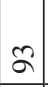 & à & $\exists$ & & 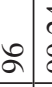 & \begin{tabular}{l}
\multirow{2}{*}{} \\
à
\end{tabular} & $\stackrel{+}{\stackrel{+}{a}}$ & $\exists$ & 2 & $\begin{array}{l}0 \\
\alpha \\
\alpha\end{array}$ & F & a & $\bar{a}$ \\
\hline ثَّ & 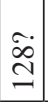 & $\begin{array}{l}\hat{\infty} \\
\stackrel{\sim}{-}\end{array}$ & 可: & 卞 & $\infty$ & 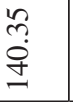 & 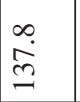 & 원 & 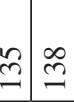 & $\begin{array}{l}\overrightarrow{\dot{n}} \\
\stackrel{n}{n}\end{array}$ & 婄 & & $\cong$ \\
\hline 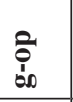 & $\begin{array}{l}\tilde{\sigma} \\
\stackrel{0}{\infty} \\
-\end{array}$ & $\Xi$ & 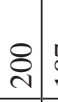 & $\stackrel{\infty}{\infty}$ & 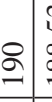 & $\begin{array}{l}n \\
\infty \\
\infty \\
\infty \\
\infty\end{array}$ & $\begin{array}{l}0 \\
\dot{0} \\
\infty \\
\infty\end{array}$ & 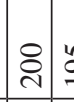 & 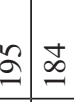 & $\underset{\infty}{\stackrel{\infty}{\infty}}$ & 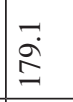 & 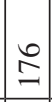 & $\Xi$ \\
\hline & $\Sigma$ & $\Sigma$ & $\Sigma$ & $\Sigma 1$ & $\Sigma$ & $\Sigma$ & $\Sigma$ & L & \begin{tabular}{l|l} 
& L
\end{tabular} & 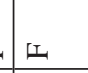 & L & a. & a. \\
\hline 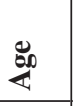 & 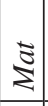 & 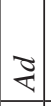 & 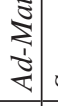 & 气ั & $\Xi$ & & & 3 & $\frac{\pi}{2}$ & & & & $\stackrel{=}{\Xi}$ \\
\hline 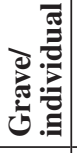 & $\begin{array}{l}2 \\
\infty \\
\infty\end{array}$ & 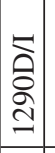 & $\begin{array}{l}\overrightarrow{0} \\
\stackrel{+}{+}\end{array}$ & & 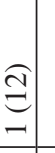 & & & 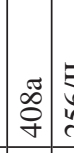 & 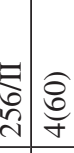 & & & & $\Xi$ \\
\hline & 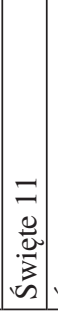 & 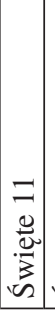 & 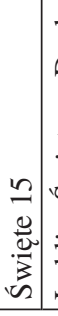 & 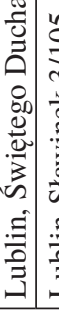 & 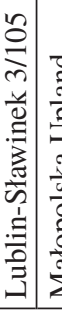 & 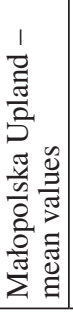 & 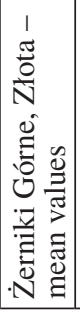 & 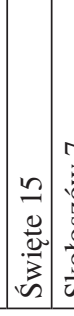 & 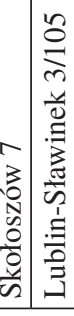 & 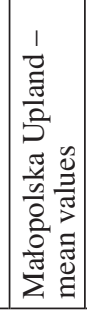 & 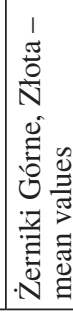 & 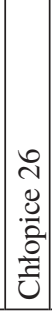 & 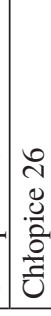 \\
\hline
\end{tabular}




\begin{tabular}{|c|c|c|c|c|c|c|c|c|c|c|c|c|c|c|c|}
\hline & 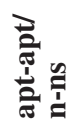 & g) & $\vec{\infty}$ & & & $\begin{array}{l}\mathfrak{n} \\
\stackrel{6}{n} \\
n\end{array}$ & 궁 & & $\begin{array}{l}n \\
n \\
n\end{array}$ & & $\begin{array}{l}\hat{y} \\
\dot{f}\end{array}$ & $\hat{q}$ & 守 & $\begin{array}{c} \pm \\
n \\
\infty \\
\infty \\
\end{array}$ & in \\
\hline & 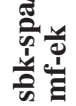 & ָָ' & & & & $\left|\begin{array}{c}0 \\
n \\
i \\
\infty\end{array}\right|$ & $\stackrel{2}{2}$ & & $\underset{\infty}{\infty}$ & & $\begin{array}{l}\infty \\
\dot{n} \\
\stackrel{n}{2}\end{array}$ & $\underset{\sim}{+}$ & 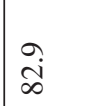 & $\begin{array}{l}\infty \\
0 \\
\dot{r} \\
i\end{array}$ & $\begin{array}{l}\infty \\
\infty \\
\infty \\
\infty\end{array}$ \\
\hline & 离 & & & & & 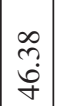 & กै & & $\begin{array}{l}n \\
\infty \\
+\end{array}$ & & $\begin{array}{l}2 \\
\infty \\
\infty \\
\dot{\alpha}\end{array}$ & î & $\hat{n}$ & \begin{tabular}{|c|}
$\infty$ \\
$\stackrel{\infty}{\infty}$ \\
$\stackrel{\infty}{f}$
\end{tabular} & $\vec{n}$ \\
\hline & 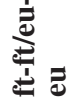 & $\stackrel{i}{i}$ & $\stackrel{?}{?}$ & $\stackrel{i}{\infty}$ & & $\begin{array}{l}n \\
0 \\
0\end{array}$ & $\stackrel{i}{i}$ & $\stackrel{0}{\stackrel{0}{\pi}}$ & $\stackrel{?}{2}$ & & $\frac{\pi}{\stackrel{1}{2}}$ & ติ่ & 8 & 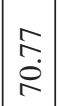 & $\stackrel{\infty}{i}$ \\
\hline & 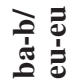 & & & $\begin{array}{l}\infty \\
\stackrel{\sigma}{\sigma}\end{array}$ & $\begin{array}{l}t \\
0 \\
\infty\end{array}$ & $\left|\begin{array}{l}\tilde{1} \\
\dot{J}\end{array}\right|$ & $\begin{array}{l}\bar{a} \\
\grave{\alpha}\end{array}$ & $\begin{array}{l}+ \\
\text { ma } \\
\text { a }\end{array}$ & 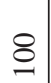 & & $\begin{array}{l}\vec{f} \\
\infty \\
\infty \\
\infty\end{array}$ & ñ & -ே: & $\left|\begin{array}{l}0 \\
+ \\
\infty \\
\infty\end{array}\right|$ & $\stackrel{\infty}{\dot{\Xi}}$ \\
\hline $\begin{array}{l}\frac{D}{0} \\
\frac{0}{0}\end{array}$ & 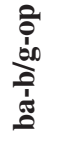 & & & $\stackrel{n}{n}$ & $\begin{array}{l}\stackrel{+}{n} \\
\stackrel{2}{*}\end{array}$ & $\left|\begin{array}{c}\mathcal{N} \\
\multirow{2}{*}{} \\
0 \\
0\end{array}\right|$ & $\ddot{n}$ & 号 & 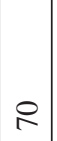 & & $\begin{array}{l}n \\
\ddot{8} \\
6\end{array}$ & $\frac{9}{\pi}$ & $\stackrel{\infty}{\gtrless}$ & $\stackrel{m}{\stackrel{n}{N}}$ & $\stackrel{9}{\stackrel{2}{ }}$ \\
\hline 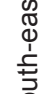 & 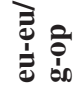 & $\stackrel{\hat{\tau}}{\circ}$ & $\stackrel{m}{N}$ & $\dddot{n}$ & $\begin{array}{l}\vec{b} \\
\infty \\
\infty\end{array}$ & $\begin{array}{l}0 \\
i \\
i\end{array} \mid$ & $\begin{array}{l}\hat{\sigma} \\
\dot{t}\end{array}$ & $\stackrel{v}{n}$ & $尺$ & กิ & $\curvearrowleft$ & $\stackrel{n}{i}$ & $\stackrel{n}{n}$ & $\mid \begin{array}{l}0 \\
\infty \\
\\
\end{array}$ & 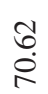 \\
\hline$\frac{0}{2}$ & 苛 & $\Sigma$ & $\Sigma$ & $\Sigma$ & $\Sigma$ & $\Sigma$ & $\Sigma$ & $\Sigma$ & 巧 & 工 & 工 & $\omega$ & L & $\sigma$. & a. \\
\hline $\begin{array}{l}\stackrel{\varrho}{0} \\
\stackrel{0}{\frac{1}{3}} \\
\frac{1}{3}\end{array}$ & $\underset{8}{8}$ & 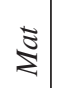 & $\underset{\pi}{\pi}$ & 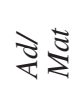 & స్ & 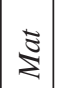 & & & 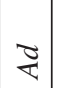 & 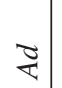 & 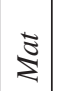 & & & 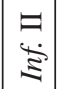 & $\begin{array}{l}= \\
\stackrel{5}{5}\end{array}$ \\
\hline $\begin{array}{l}\frac{0}{2} \\
\frac{1}{10} \\
0 \\
0 \\
\frac{0}{0} \\
0 \\
0\end{array}$ & 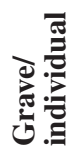 & $\frac{0}{\infty}$ & 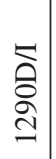 & $\begin{array}{l}0 \\
\stackrel{+}{+}\end{array}$ & & $\stackrel{\overparen{\Xi}}{\Xi}$ & & & 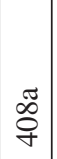 & 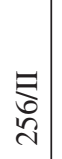 & $\underset{\hat{\sigma}}{\stackrel{\theta}{\sigma}}$ & & & $\Xi$ & $\Xi$ \\
\hline 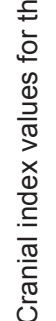 & 華 & 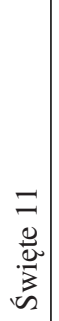 & 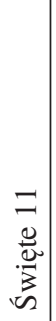 & $\begin{array}{l}n \\
\frac{0}{0} \\
\frac{0}{3} \\
n\end{array}$ & 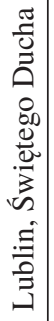 & 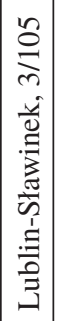 & 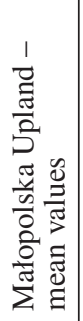 & 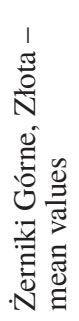 & $\begin{array}{c}n \\
0 \\
\frac{0}{2} \\
3 \\
n\end{array} \mid$ & 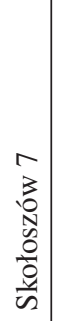 & 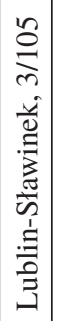 & 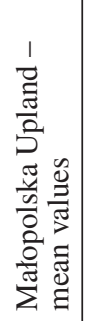 & 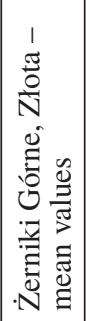 & 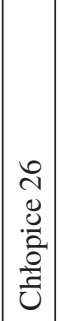 & 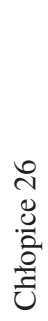 \\
\hline
\end{tabular}




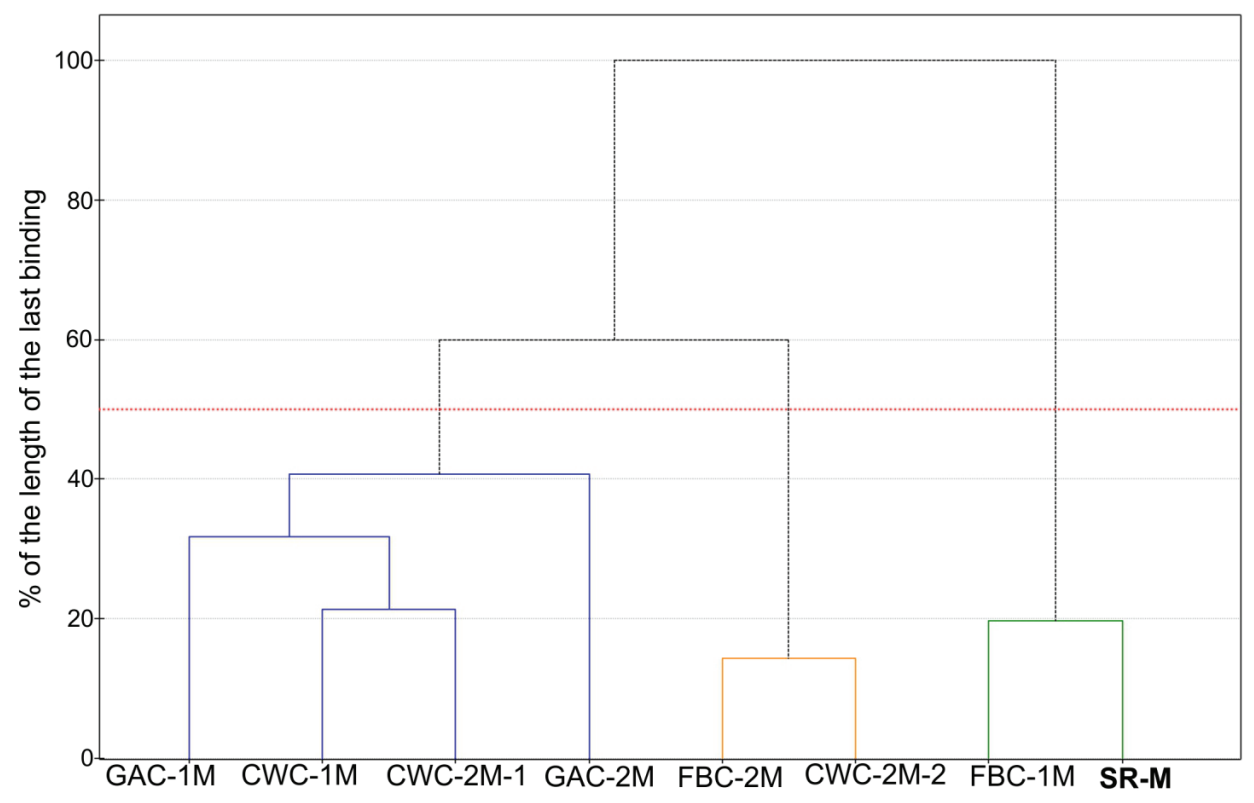

Fig. 2. Hierarchical cluster analysis of male crania (grouping by Ward's method) from southeastern Poland at the end of the Neolithic

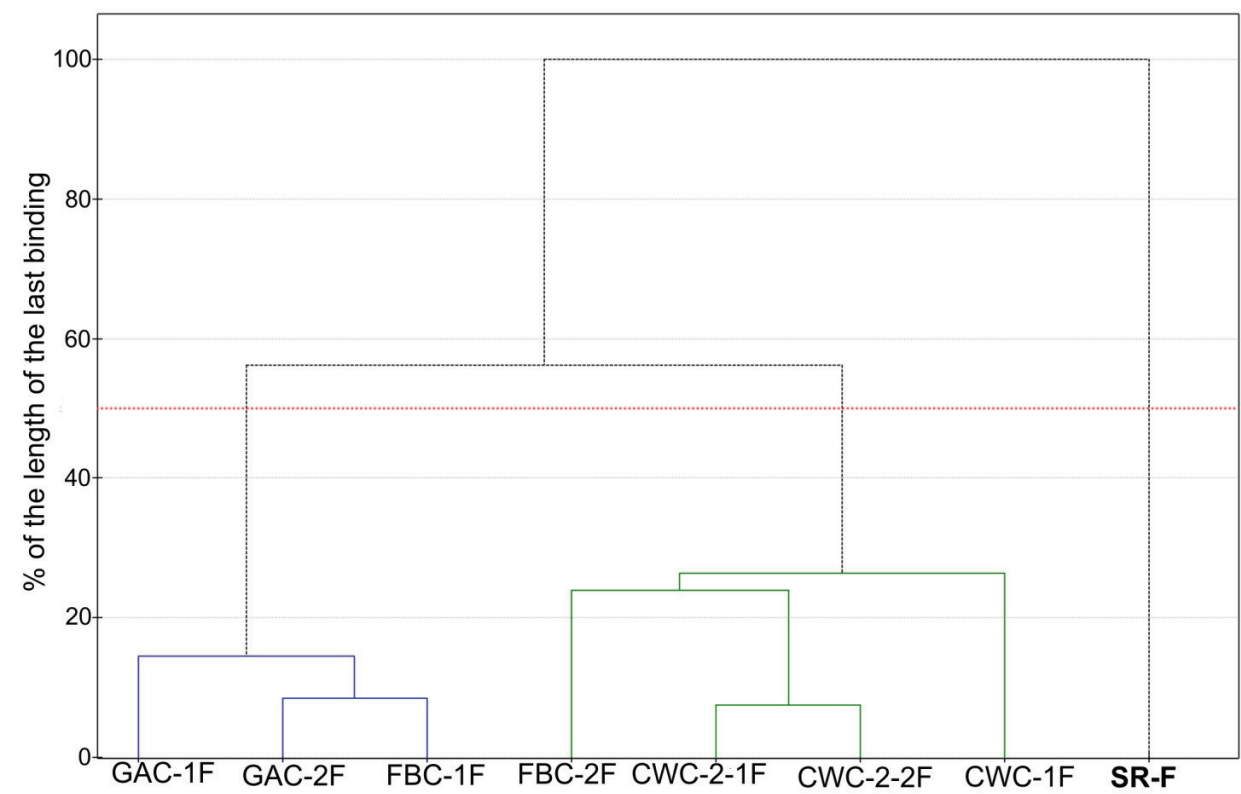

Fig. 3. Hierarchical cluster analysis of female crania (grouping by Ward's method) from southeastern Poland at the end of the Neolithic 
generalization of the results so obtained to the entire population occupying the area at issue.

The measurements and cranial indices were used to analyse differences between cranial data for CWC people occupying south-eastern Poland and those for Neolithic sets from the same area. The analysis was performed using the PQStat software through hierarchical cluster analysis of male and female sets, calculation of Euclidean distances, and data grouping using Ward's method (Figs. 2 and 3 ). By reason of the degree of skulls preservation, data obtained for neurocrania were used for the statistical analysis. The measurements included cranial length ( $g$-op), cranial breadth $(e u-e u)$, forehead breadth $(\mathrm{ft}-\mathrm{ft})$ and cranial indexes such as the main cranial index (eu-eu/g-op x100) and the forehead width index (ft-ft/ eu-eu x100). Mean values of measurements and indices for the skulls of CWC, Funnel Beaker culture (FBC) and Globular Amphora Culture (GAC) individuals, unearthened at the sites located in the Rzeszów Foothills and Lower San River Valley region (SR), Lublin Upland (FBC-1, GAC-1, CWC-1) as well as Małopolska Upland (FBC-2, GAC-2, CWC-2) were compared, with the division between materials published by E. Haduch [Haduch 2008] (CWC-2-1) and those published by M. Krenz-Niedbała [1999; 2000] (CWC-2-2) kept.

Cranial dendrograms for both male and female sets show three clusters; provided, however, that the female crania from the examined region form a separate group, while the male crania group with crania of the FBC individuals from the Lublin Upland. Such a result may be, however, accidental as the sample is small and gives clearly higher maximum cranial lengths. It should be noted that both dendrograms show the GAC individuals within one cluster, with distances between particular sets being small, especially for the females.

Tables 6 and 7 collate measurements of maximum cranial lengths (M1) of limb bones, and estimated intravital body heights of buried individuals, using regression equations developed by Formicola and Franceschi [1996]. The graph (Fig. 4) shows reconstructed intravital body heights for CWC people occupying south-eastern Poland, with trend lines for male and female sets.

Adult body height varies in both male and female sets. As far as the Rzeszów Foothills and Lower San River Valley are concerned, adult body height is 161.3$-175 \mathrm{~cm}$ for males and 156.7-163.1 cm for females. For most of the males, these values are within the range determined for other CWC individuals of south-eastern Poland. Body height of two females, one buried at Święte, site 20 (grave 43), and the other buried at Mirocin, site 27 (grave 360), somewhat exceeds the value determined for other CWC females, falling within the male height range. It proves the reduced level of sexual dimorphism and indirectly indicates diversified living conditions of buried individuals. 
T a b l e 6

Measurements of maximum lengths of long bones (M1: $\mathrm{mm})$ in Corded Ware culture adults

\begin{tabular}{|c|c|c|c|c|c|c|c|c|c|c|}
\hline \multirow[t]{2}{*}{ Site } & \multirow[t]{2}{*}{ Grave } & \multirow[t]{2}{*}{ Sex } & \multicolumn{2}{|c|}{ Humerus } & \multicolumn{2}{|c|}{ Radius } & \multicolumn{2}{|c|}{ Femur } & \multicolumn{2}{|c|}{ Tibia } \\
\hline & & & $\mathrm{R}$ & $\mathrm{L}$ & $\mathrm{R}$ & $\mathrm{L}$ & $\mathrm{R}$ & $\mathrm{L}$ & $\mathrm{R}$ & $\mathrm{L}$ \\
\hline Mirocin 24 & $110 / 1$ & $\mathrm{M}$ & & & & 253 & 471 & & & 385 \\
\hline Mirocin 27 & $360 / 1$ & M & & & & & & & & 364 \\
\hline Święte 11 & 876 & M & 336 & 329 & & 267 & & & 386 & \\
\hline Święte 11 & 1134 & M & & & & 261 & & & 376 & \\
\hline Święte 11 & $1290 \mathrm{D}$ & M & 324 & 318 & 254 & & 460 & 460 & 381 & 380 \\
\hline Święte 15 & $408 b$ & M & 311 & 308 & 232 & 233 & 422 & 420 & 352 & 350 \\
\hline Święte 20 & $43 / I$ & M & & & & & 454 & 455 & & \\
\hline Skołoszów & 620 & M & 310 & 310 & & 234 & 452 & 452 & 370 & 366 \\
\hline Szczytna 6 & 56 & $\mathrm{M}$ & & 346 & & & 489 & 489 & 399 & 410 \\
\hline $\begin{array}{l}\text { Lublin, Świętego } \\
\text { Ducha }\end{array}$ & & M & & & & & 485 & & 402 & 403 \\
\hline $\begin{array}{l}\text { Lublin-Sławinek } \\
\text { 3/105 }\end{array}$ & $1(12)$ & M & 320 & 317 & 243 & 241 & 441 & 447 & 367 & 366 \\
\hline $\begin{array}{l}\text { Małopolska Up- } \\
\text { land - mean values }\end{array}$ & & $\mathrm{M}$ & 318.7 & & 242.4 & & 440.2 & & 372. & \\
\hline $\begin{array}{l}\text { Żerniki Górne, } \\
\text { Złota - mean } \\
\text { values }\end{array}$ & & M & 323.8 & & 246.6 & & 450.1 & & 373. & \\
\hline Mirocin 27 & $360 / 2$ & $\mathrm{~F}$ & 326 & & & 249 & 452 & 455 & 384 & 381 \\
\hline Święte 15 & 173 & $\mathrm{~F}$ & & & & & 431 & 428 & 346 & 347 \\
\hline Święte 20 & 43/II & $\mathrm{F}$ & & & & & 443 & 445 & & \\
\hline $\begin{array}{l}\text { Lublin-Sławinek } \\
3 / 105\end{array}$ & $4(60)$ & $\mathrm{F}$ & 312 & 310 & & & & & 370 & 370 \\
\hline $\begin{array}{l}\text { Małopolska Up- } \\
\text { land - mean values }\end{array}$ & & $\mathrm{F}$ & 291.7 & & 207.5 & & 411.8 & & 341. & \\
\hline $\begin{array}{l}\text { Żerniki Górne, } \\
\text { Złota - mean } \\
\text { values }\end{array}$ & & $\mathrm{F}$ & 302.6 & & 228.4 & & 420.3 & & 347. & \\
\hline
\end{tabular}

In recent decades, archaeological research has substantially added to the discovery of numerous skeletal remains of CWC individuals from the Rzeszów Foothills. The remains represent just a part of the larger population that once occupied the region. Presented above, the anthropological picture is relatively incomplete due to poor preservation of skeletons, including specifically considerable second- 
Estimated intravital body heights $[\mathrm{cm}]$ of Corded Ware culture people

\begin{tabular}{|c|c|c|c|c|c|c|c|c|c|c|}
\hline \multicolumn{11}{|c|}{ Male } \\
\hline \multirow[t]{2}{*}{ Site } & \multirow[t]{2}{*}{ Grave } & \multicolumn{2}{|c|}{$\begin{array}{l}\text { Humerus } \\
\mathbf{3 , 3 1 \times H + 6 0 , 8 7}\end{array}$} & \multicolumn{2}{|c|}{\begin{tabular}{|l|} 
Radius \\
$\mathbf{3 , 6 5 x R + 7 5 , 5 7}$ \\
\end{tabular}} & \multicolumn{2}{|c|}{$\begin{array}{l}\text { Femur } \\
2,23 \times F+65,9 \\
\end{array}$} & \multicolumn{2}{|c|}{\begin{tabular}{|l|} 
Tibia \\
$\mathbf{2 , 4 7 x T}+\mathbf{7 4 , 8 4}$ \\
\end{tabular}} & \multirow[t]{2}{*}{$\begin{array}{l}\text { Mean } \\
\text { value }\end{array}$} \\
\hline & & $\mathrm{R}$ & $\mathrm{L}$ & $\mathrm{R}$ & $\mathrm{L}$ & $\mathrm{R}$ & $\mathrm{L}$ & $\mathrm{R}$ & $\mathrm{L}$ & \\
\hline Mirocin 24 & $110 / 1$ & & & & 167.9 & 170.9 & & & 169.9 & 169.6 \\
\hline Mirocin 27 & $360 / 1$ & & & & & & & & 164.7 & 164.7 \\
\hline Święte 11 & 876 & 172.1 & 169.8 & & 173 & & & 170.2 & & 171.3 \\
\hline Święte 11 & 1134 & & & & 170.8 & & & 167.7 & & 169.3 \\
\hline Święte 11 & $1290 \mathrm{D}$ & 168.1 & 166.1 & 168.3 & & 168.5 & 168.5 & 168.9 & 168.7 & 168.2 \\
\hline Święte 15 & $408 \mathrm{~b}$ & 163.8 & 162.8 & 160.3 & 160.6 & 160 & 159.6 & \begin{tabular}{|l|}
161.8 \\
\end{tabular} & 161.3 & 161.3 \\
\hline Święte 20 & $43 / I$ & & & & & 167.1 & 167.4 & & & 167.3 \\
\hline Skołoszów 7 & 620 & 163.5 & 163.5 & & 161 & 166.7 & 166.7 & 166.2 & 165.2 & 164.7 \\
\hline Szczytna 6 & 56 & & 175.4 & & & 174.9 & 174.9 & 173.4 & 176.1 & 175 \\
\hline $\begin{array}{l}\text { Lublin, } \\
\text { Swiętego Ducha }\end{array}$ & & & & & 174 & & & 174 & 174 & 174 \\
\hline $\begin{array}{l}\text { Lublin- } \\
\text { Sławinek 3/105 }\end{array}$ & 1(12) & 167 & 166 & 164 & 164 & 164 & 166 & 165 & 165 & 165 \\
\hline $\begin{array}{l}\text { Małopolska } \\
\text { Upland - mean } \\
\text { values }\end{array}$ & & \multicolumn{2}{|l|}{171.5} & \multicolumn{2}{|l|}{166} & \multicolumn{2}{|l|}{169.6} & \multicolumn{2}{|l|}{169.4} & 169.1 \\
\hline $\begin{array}{l}\text { Żerniki Górne, } \\
\text { Złota - mean } \\
\text { values }\end{array}$ & & \multicolumn{2}{|l|}{166} & \multicolumn{2}{|l|}{164} & \multicolumn{2}{|l|}{164} & \multicolumn{2}{|l|}{167} & 165 \\
\hline \multicolumn{11}{|c|}{ Female } \\
\hline \multirow[t]{2}{*}{ Site } & \multirow[t]{2}{*}{ Grave } & \multicolumn{2}{|c|}{\begin{tabular}{l|} 
Humerus \\
$2,88 \times H+69,35$
\end{tabular}} & \multicolumn{2}{|c|}{$\begin{array}{l}\text { Radius } \\
2,69 \times R+92,83 \\
\end{array}$} & \multicolumn{2}{|c|}{\begin{tabular}{|l|} 
Femur \\
$2,35 x F+56,63$ \\
\end{tabular}} & \multicolumn{2}{|c|}{\begin{tabular}{|l|} 
Tibia \\
$\mathbf{2 , 4 6 x T + 7 0 , 5 7}$ \\
\end{tabular}} & $\begin{array}{l}\text { Mean } \\
\text { value }\end{array}$ \\
\hline & & $\mathrm{P}$ & $\mathrm{L}$ & $\mathrm{P}$ & $\mathrm{L}$ & $\mathrm{P}$ & $\mathrm{L}$ & $\mathrm{P}$ & $\mathrm{L}$ & \\
\hline Mirocin 27 & $360 / 2$ & 163.2 & & & 159.8 & 162.9 & 163.6 & 165 & 164.3 & 163.1 \\
\hline Święte 15 & 173 & & & & & 157.9 & 157.2 & 155.7 & 155.9 & 156.7 \\
\hline Święte 20 & $43 / \mathrm{II}$ & & & & & 160.7 & 161.2 & & & 161 \\
\hline $\begin{array}{l}\text { Lublin- } \\
\text { Sławinek 3/105 }\end{array}$ & $4(60)$ & 159 & 159 & & & & & 162 & 162 & 160 \\
\hline $\begin{array}{l}\text { Małopolska } \\
\text { Upland - mean } \\
\text { values }\end{array}$ & & \multicolumn{2}{|l|}{153.4} & \multicolumn{2}{|l|}{148.6} & \multicolumn{2}{|l|}{153.4} & \multicolumn{2}{|l|}{154.6} & 153 \\
\hline $\begin{array}{l}\text { Żerniki Górne, } \\
\text { Złota - mean } \\
\text { values }\end{array}$ & & \multicolumn{2}{|l|}{156} & \multicolumn{2}{|l|}{154} & \multicolumn{2}{|l|}{155} & \multicolumn{2}{|l|}{156} & 156 \\
\hline
\end{tabular}




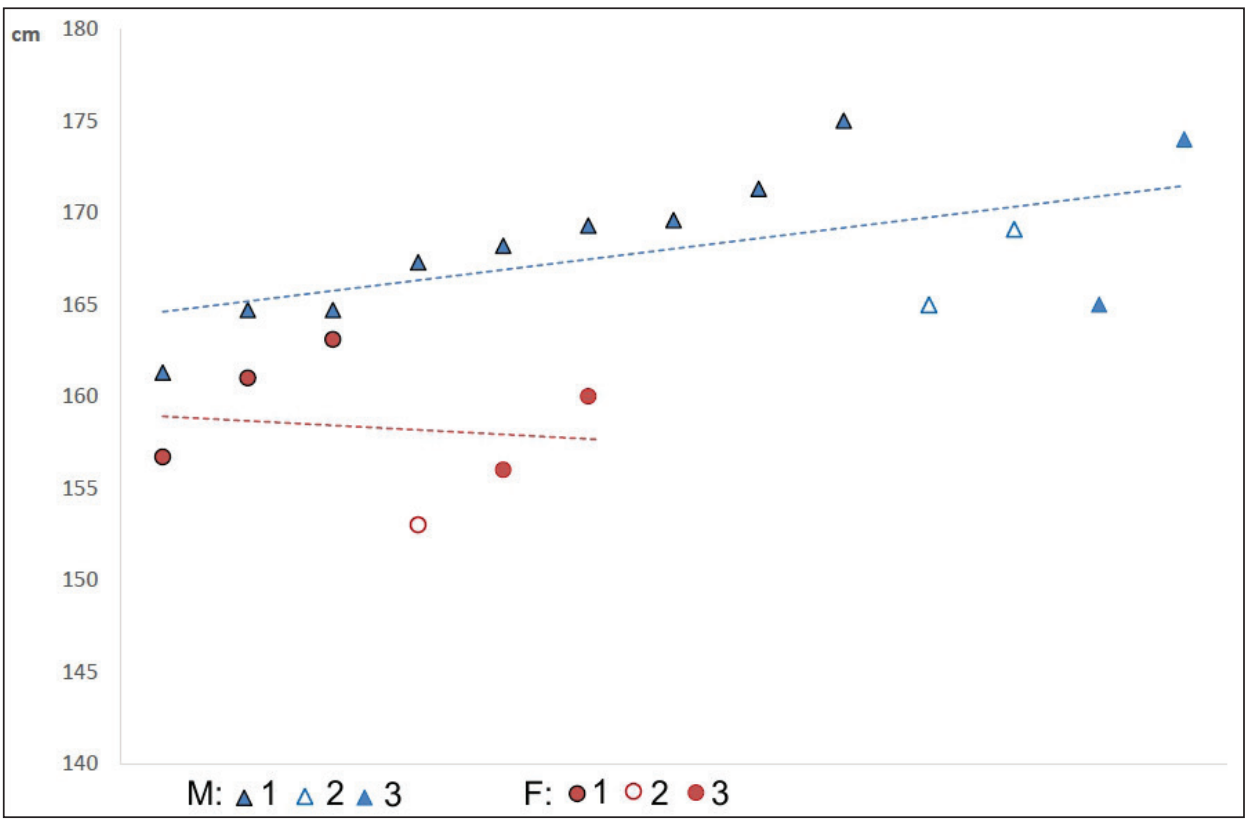

Fig. 4. Estimated intravital body heights of Corded Ware culture people from south-eastern Poland: M (male): 1 - Rzeszów Foothills and Lower San River Valley, 2 - Małopolska Upland, 3 - Lublin Upland; F (female): 4 - Rzeszów Foothills and Lower San River Valley, 5 - Małopolska Upland, 6 - Lublin Upland

ary damages to crania. In most of the cases, however, precise determination of age and sex of buried individuals was possible, providing a basis for reflection on burial customs and allowing for interpretation of isotopic results [Belka et al. 2018; Werens et al. 2018]. Although scarce, the anthropometric data make it possible to conclude that both male and female crania are dolichomorphic: a similar phenomenon has been already observed on the Małopolska Upland [Haduch 2008] as well as in present-day Ukraine [Konduktorova 1978] and on the Czech territory [Chochol 1967]. 


\section{REFERENCES}

Belka Z., Dopieralska J., Szczepanek A., Jarosz P.

2018 Human mobility in the Final Eneolithic population of Święte, Jarosław District, south-eastern Poland: evidence from strontium isotope data. Baltic-Pontic Studies 23: 246-258.

Chochol J.

1967 Zur Anthropologie der böhmischen Schnurkeramiker, In: M. Buchvaldek, Die Schnurkeramik in Böhmen. Acta Universitas Carolinae Philosophica et Historica, Monographia 19, 207-216. Praha.

Dąbrowski P.

2012 Analiza antropologiczna szczątków kostnych ze stanowiska archeologicznego Święte (St. 20), gm. Radymno. In: K. Nowaczyk L. Nowaczyk (Eds) Opracowanie wyników ratowniczych badań archeologicznych na stanowisku Święte 20 (azp 106-84/132), gm. Radymno, pow. jarosławski, woj. podkarpackie [manuscript]. Warszawa.

Formicolla V., Franceschi M.

1996 Regression equations for estimating stature from long bones Early Holocene European samples. American Journal of Physical Anthropology 100: 83-88.

Haduch E.

2008 Ludność kultury ceramiki sznurowej na obszarze Wyżyny Małopolskiej. In: K. Kaczanowski (Ed.) 100-lecie Zakładu Antropologii UJ, Księga Jubileuszowa, 197-206. Kraków.

Konduktorowa T.C.

1978 Antropologicheskiy typ ludey kultur shnurovoy keramiki Ukrainy. Voprosy Antropologii 59: 3-23.

Koperski A.

1973 Grób kultury ceramiki sznurowej w Przemyślu przy ul. Pstrowskiego. Sprawozdania Archeologiczne 25: 79-82.

Kozak-Zychman W.

2011 Materiały kostne z pochówków odkrytych w gminie Radymno - analiza antropologiczna. In: Święte 11 stanowisko w gminie Radymno, woj. podkarpackie [manuscript]. Warszawa. 
Krenz-Niedbała M.

1999 Differentiation of the Biological Structure in the Populations of the Corded Ware Culture and Lengyel Culture. In: D. Jankowska, M. Krenz-Niedbała, J. Piontek, J. Wierzbicki (Eds) Biological and cultural consequences of the transition to agriculture in Central Europe. Monografie Instytutu Antropologii UAM 4, 45-59. Poznań.

2000 Biologiczne i kulturowe skutki neolityzacji w populacjach ludzkich na ziemiach polskich. Monografie Instytutu Antropologii UAM 8. Poznań.

Rogóż J.

2017 Analiza antropologiczna szkieletów kultury ceramiki sznurowej i domniemanej mierzanowickiej ze Szczytnej na stanowiskach 5 i 6, pow. Jarosław. In: P. Jarosz, J. Machnik (Eds) Nekropolie ludności kultury ceramiki sznurowej z III tysiaclecia przed Chr.w Szczytnej na Wysoczyźnie Kańczuckiej. Via Archeologica Ressoviensia 12, 191-214. Rzeszów.

Rutka H.

2013 Analiza antropologiczna materiału kostnego ze stanowiska Święte 15/127/106-84 azp In: Święte 15/127/106-84 azp stanowisko w gminie Radymno, woj. podkarpackie [manuscript]. Warszawa.

Szczepanek A.

2004 Analiza antropologiczna szkieletu z grobu kultury ceramiki sznurowej z Przemyśla, stan. 81 [manuscript]. Kraków.

2012 Analiza antropologiczna ludzkich szczątków z obiektu 11 w Chłopicach, st. 26. In: J. Lepiejza, Opracowanie wyników badań wykopaliskowych na stanowisku $\mathrm{nr} 26$ w Chłopicach [manuscript]. Rzeszów.

2013 a Analiza antropologiczna szkieletów ze stanowiska 42 w Rozbórzu [manuscript]. Rzeszów.

2013 b Analiza antropologiczna szkieletów ze stanowiska 7 w Skołoszowie [manuscript]. Rzeszów.

2016 Ludność kultury amfor kulistych i kultury ceramiki sznurowej na Wyżynie Lubelskiej w świetle badań antropologicznych. In: P. Jarosz, J. Libera, P. Włodarczak (Eds) Schyłek neolitu na Wyżynie Lubelskiej, 493-508. Kraków.

2017 Analiza antropologiczna ludzkich szczątków z grobu kultury ceramiki sznurowej nr 220 w Szczytnej, pow. Jarosław, stanowisko 5. In: P. Jarosz, J. Machnik (Eds) Nekropolie ludności kultury ceramiki sznurowej z III tysiąclecia przed Chr. w Szczytnej na Wysoczyźnie Kańczuckiej. Via Archeologica Ressoviensia 12, 215-220. Rzeszów.

2019 Analiza antropologiczna szkieletów ludności kultury ceramiki sznurowej z grobów w Mirocinie, stanowiska 24 i 27, pow. Przeworsk In: P. Jarosz, 
J. Machnik, A. Szczepanek (Eds) Nekropolie ludności kultury ceramiki sznurowej z III tysiaclecia przed Chr. w Mirocinie na Wysoczyźnie Kańczuckiej, Via Archaeologica Ressoviensia, 313-323. Rzeszów.

Walker P. L., Bathurst R. R., Richman R., Gjerdrum T., Andrushko V. A.

2009 The causes of porotic hyperostosis and cribra orbitalia: A reappraisal of the iron deficiency anemia hypothesis. American Journal of Physical Anthropology 139(2): 109-125.

Werens K., Szczepanek A., Jarosz P.

2018 Light stable isotope analysis of diet in Corded Ware culture communities: Święte, Jarosław District, south-eastern Poland. Baltic-Pontic Studies 23: 229-245. 